\title{
Primary Pulmonary Sequestration With Secondary Hamartomatosis Change
}

\author{
Changhu Liang ${ }^{1}$; Shifeng Xu ${ }^{2, *}$; Bin Zhao ${ }^{1}$; Guoyuan Ma ${ }^{3}$; Yinglin Du ${ }^{4}$ \\ ${ }^{1}$ Shandong Medical Imaging Research Institute, Affiliated to Shandong University, Jinan, P. R. China \\ 2 Department of Surgery, Provincial Hospital, Affiliated to Shandong University, Jinan, P. R. China
${ }^{3}$ Department of Thoracic Surgery, Provincial Hospital, Affiliated to Shandong University, Jinan, P. R. China

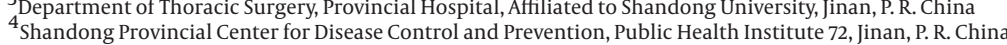 \\ ${ }^{*}$ Corresponding author: Shifeng Xu, Department of Surgery, Provincial Hospital, Affiliated to Shandong University, Jinan, P. R. China. Tel: +86-53168776931, Fax: +86- \\ 53187938911, E-mail: ShifengXu123456@163.com
}

Received: April 15, 2013; Revised: February 27, 2014; Accepted: May 17, 2014

\begin{abstract}
The radiologic features of intralobar pulmonary sequestration (ILPS) have been describe and include the identification of a feeding systemic artery with venous drainage through pulmonary veins. Primary sequestration associated with typical hamartoma signs is really rare and has been described only once. We describe a patient with ILPS whose radiographic findings were unusual for two reasons. First, computed tomography (CT) demonstrated a bulky mass in the pulmonary sequestration. The size of lesion and histopathology made it an unusual presentation. Final histology study demonstrated pulmonary hamartoma with predominantly adipose and cartilage differentiation, which is an unusual complication originated from ILPS. Another sign also explains the second unusual feature, intracranial cholesteatoma, occurring concurrently with ILPS.
\end{abstract}

Keywords: Pulmonary Sequestration; Hamartoma; Syndrome

\section{Introduction}

Intralobar pulmonary sequestration (ILPS) with an anomalous systemic arterial blood supply represents a mass of nonfunctioning parenchyma surrounded by visceral pleura $(1,2)$. Unlike extralobar sequestration, it is rarely associated with other developmental abnormalities. Radiographically demonstrated calcification in the ILPS has been reported in some case series, but primary sequestration associated with typical hamartoma signs is really rare and has been described only once $(3,4)$. In this report, we present a rare case of giant hamartoma originated from primary ILPS in a 39-year-old woman who had intracranial cholesteatoma.

\section{Case Presentation}

A 39-year-old woman with an abnormal mass in the right cerebellopontine angle was referred to neurosurgery department of our hospital. A giant mass in the right thoracic cavity was detected accidentally by X-ray in a routine preoperative screening process. Physical examination demonstrated some loss of breathing sounds in the right base. Subsequent thoracic computed tomography scan demonstrated a complex lesion (size $15.1 \times 12.3 \times$ $15.7 \mathrm{~cm}$ ) occupying more than two-thirds of the thoracic cavity on the right side. The blood supply to the area of the lesion was from a systemic artery arising from the abdominal aorta, with venous drainage through the inferior pulmonary vein, suggesting an ILPS (Figure $1 \mathrm{~A}-1 \mathrm{C}$ ). She had no history of weight loss, pneumonia, hemoptysis and dyspnea. After careful consultation, a right temporal craniotomy for intracranial lesion was performed. The intracranial mass was completely excised and histopathological study revealed the tumor as cholesteatoma. The patient's postoperative recovery was uneventful.

Three months later, an open thoracic surgery for the intrathoracic lesion was performed under general anesthesia. Intraoperatively, lesion appeared hypervascular, because of abundant systemic vascularization (Figure $2 \mathrm{~A}$ ). The massive lesion was compressing the right lung and there was no communication between the tracheobronchial tree and sequestered lesion. Based on the findings of 3D CT angiography, we explored the aberrant artery at the bottom of the chest and carefully ligated it with an absorbable silk suture. After the pulmonary ligament had been completely divided, the lower pulmonary vein was found to be widened. The lesion was adherent to adjacent pulmonary vein branches and was not easily dissected. To avoid major intraoperative blood loss, a right lower lobectomy was performed following resection of the sequestered lesion. Pathologic analysis showed a predominant fatty differentiation with fibroblastic, chondroid and osseous differentiation making the rest (Figure 2B 2D). The residual lung appeared anatomically normal following resection of the sequestration. The postoperative recovery was uneventful and the patient was discharged from the hospital on the eighth postoperative day.

Copyright (C) 2015, Tehran University of Medical Sciences and Iranian Society of Radiology. This is an open-access article distributed under the terms of the Creative Commons Attribution-NonCommercial 4.0 International License (http://creativecommons.org/licenses/by-nc/4.0/) which permits copy and redistribute the material just in noncommercial usages, provided the original work is properly cited. 

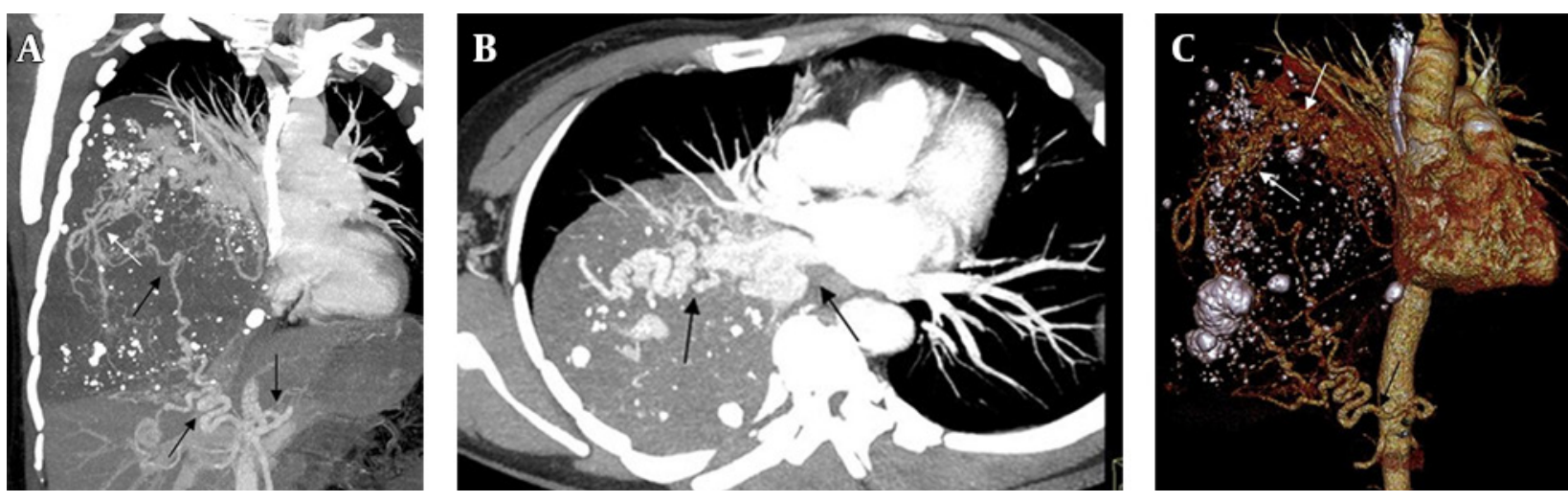

Figure 1. Preoperative computed tomographic angiography in a 39-year-old woman. Mean intensity projection (A,B) and volume rendering (C) images demonstrate a circuitous systemic artery (black arrow) arising from the abdominal aortic with venous drainage into the left atrium through widened, tortuous pulmonary vein (white arrow).
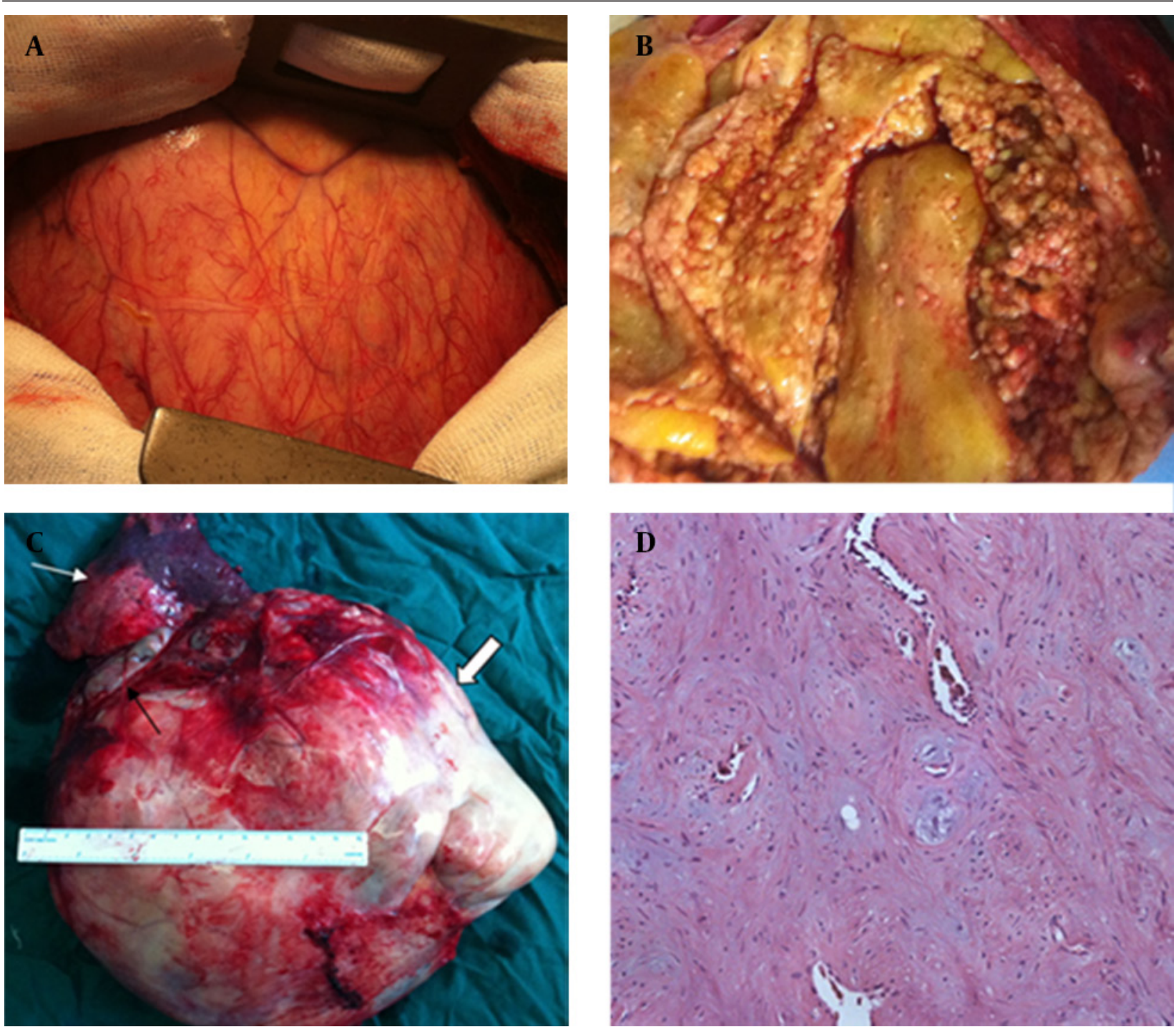

Figure 2. A, intraoperative view of the giant lesion and the hypervascular surface. B, the internal components of the lesion. C, gross specimen photograph of the lesion: right lower lobe (white arrow) and lesion (thick arrow) with the draining vein (black arrow). D, pathology (200 $\mu \mathrm{m}$ ): predominant fatty differentiation, with intermixed chondroid and osseous areas. 


\section{Discussion}

ILPS is usually diagnosed later than extralobar sequestration (ELS), being found in childhood or adulthood when the patient presents with an infection (5). Up to $15 \%$ of patients, particularly in adult population, are asymptomatic when the sequestration is discovered incidentally (6). In our case, the sequestration surrounded by visceral pleura, characteristically receives its blood supply from a systemic artery with venous drainage through pulmonary veins and was consistent with ILPS.

The lesions of ILPS may be solid, fluid or hemorrhagic or may contain mucus. Primary sequestration associated with typical hamartoma signs is really rare. In our case, CT showed a mass containing numerous round calcifications. Final histology was pulmonary hamartoma with predominantly adipose and cartilage differentiation, which is an unusual complication originated from primary sequestration. This case represents an extremely rare variation of pulmonary sequestration. The size of lesion and histopathology made it an unusual presentation.

Pulmonary hamartoma is a rare lung neoformation, usually symptomless and discovered incidentally, of a probable dysontogenetic origin with prevailing cartilaginous tissue and adult onset age (7). In a series of 24 patients, Gabrail and Zara (8) concluded that pulmonary hamartomas are frequently accompanied by other developmental abnormalities and benign tumors. However, the entity described (pulmonary hamartoma syndrome) seems to be distinct from well-established multiple hamartoma syndrome (Cowden's syndrome). Many interesting features characterize the case report: histopathological nature of the pulmonary hamartoma, the typical vascular structure related to intrapulmonary sequestra- tion and intracranial cholesteatoma as shown by neurosurgery operation.

The pathophysiology of the association between intrapulmonary sequestration and pulmonary hamartoma is unclear. The combination of sequestration, hamartoma and cholesteatoma may be only an incidental combination, but this could indicate both possibilities as hamartomatosis change on underlying sequestration or sequestration associated with hamartomatosis syndrome.

\section{Authors' Contributions}

All authors contributed extensively to the work presented in this paper.

\section{References}

1. Miyake H, Hori Y, Takeoka H, Takuma M, Kawagoe T, Mori H. Systemic arterial supply to normal basal segments of the left lung: characteristic features on chest radiography and CT. AJR Am J Roentgenol.1998;171(2):387-92.

2. Fabre D, Rohnean A, Fadel E, Dartevelle PG. Giant aneurysmal dilation of an intralobar pulmonary sequestration artery. Eur $J$ Cardiothorac Surg. 2009;36(2):413-4.

3. Coblentz CL, Chen JT, Bernhardt P, Roggli V. Calcified intralobar pulmonary sequestration. Can Assoc Radiol J.1988;39(4):290-2.

4. Zuskin L, Iamkovoi SD, Kolesnik VI, Safonova AI. [A case of preoperative diagnosis of intrapulmonary sequestration associated with adenoma-like hamartoma]. Grud Serdechnososudistaia Khir. 1990(5):75-7.

5. Van Raemdonck D, De Boeck K, Devlieger H, Demedts M, Moerman P, Coosemans W, et al. Pulmonary sequestration: a comparison between pediatric and adult patients. EurJ Cardiothorac Surg. 2001;19(4):388-95.

6. Schena S, Crabtree TD, Zoole JB, Patterson GA. Intralobar pulmonary sequestration associated with an aneurysmal aberrant aortic branch. J Thorac Cardiovasc Surg. 2007;134(2):535-6.

7. Panzini L, Potalivo S, Saed G, Rapisarda M, Bisetti A. Pulmonary hamartoma. A rare case report. Panminerva Med.1999;41(4):359-62.

8. Gabrail NY, Zara BY. Pulmonary hamartoma syndrome. Chest. 1990;97(4):962-5. 